\title{
Meeting report: RNA-mediated gene regulation
}

\author{
Nam-Soo Kim \\ Received: 10 September 2012 / Accepted: 14 September 2012 / Published online: 10 October 2012 \\ (C) The Genetics Society of Korea and Springer 2011
}

The $67^{\text {th }}$ Annual Meeting of the Korean Association of Biological Sciences was held on August 16-17 at Chungnam National University in Daejun, Korea. Participants presented more than 430 posters and 70 oral presentations. The meeting was sponsored by the participating societies: the Korean Society Integrative Biology, the Korean Society of Limnology, the Ecological Society of Korea, the Korean Society of Biological Education, the Korean Society of Systematic Zoology, and the Genetics Society of Korea.

The meeting featured two plenary lectures. The first plenary speaker was Prof. John M. Heraty from the Department of Entomology at the University of California in Riverside, California, USA. The topic of his presentation was "Chalcidoid wasps and the interface of systematics and biology". He presented a comprehensive overview of his field collections, gathered over a 30-year period, and natural history to explain the evolution of the Chalcidoidea. His lecture integrated morphological and molecular methods into systematics, and demonstrated how future researchers can avoid taking too narrow an approach to taxonomy. The integrated approach will surely improve our understanding of the taxonomy, classification, and evolution of insects. The second plenary lecturer was Prof. Gynheung An, a professor at Kyunghee University, Korea. The topic of his presentation was "Functional genomics approaches for studying flowering time". He started his lecture with the origin of rice and the surprising finding of 13,000-year-old rice seeds in Korea: the oldest rice seeds in the world. Flowering is a very interesting and important biological event because we all depend on the energy produced by plants. Unlike the sexual cells that are secluded in an early embryonic stage in animals, apical meristem cells readily respond to environmental cues and give rise reproductive cells via flowering. Rice flowers when the days become shorter with the changing of the seasons. In order

Nam-Soo Kim $(\bowtie)$

Editor-in-Chief, Genes \& Genomics

Department of Molecular Bioscience, Kangwon National University, Chuncheon 200-701, Korea

e-mail: kimnamsu@kangwon.ac.kr to isolate the genes involved in flowering, An's group generated hundreds of thousands of T-DNA insertion mutant populations. By analyzing the mutants, his group found three different pathways that suppress flowering under long-day conditions and two pathways that induce flowering under short-day conditions. Epigenetic regulators control the flowering pathways, and Dr. An gave a detailed description of the role of microRNA 172 in suppressing AP2-family transcripts.

The recent explosion in genomics research revealed that RNAs play more significant roles in evolution than simply being the intermediary molecules of information flow. Biology's central dogma, the flow of genetic information from DNA to RNA to protein, is now obsolete. RNAs can be reverse-transcribed into DNA by reverse transcriptase. Moreover, most of the RNAs transcribed from the genome actually do not make proteins, and these noncoding RNAs instead function in gene-regulatory networks that underlie much of the genetic complexity in eukaryotes (Dennis, 2002; Mattick and Makunin, 2006). The noncoding RNAs make up as much as $98 \%$ of the transcriptional output of the human genome. It is an appealing idea that RNAs function in cellular activities because the total numbers of genes are not much different among the genomes of eukaryotic organisms. Higher organisms may use functional RNAs to generate and exploit novel combinations of conserved protein domains, thus creating the vast complexity found among the higher eukaryotic species. Because of the increasing focus on RNAs in gene regulation and evolution, we had a symposium on RNA-mediated gene regulation. The symposium consisted of two sessions of symposia; posttranscriptional regulation and RNA metabolism.

In the first session, Posttranslational regulation, three speakers gave talks. The first speaker was Prof. Haihong Shen from the Gwangju Institute of Science and Technology (GIST), Korea. The title of her talk was "The U2AF35-related protein Urp contacts the 3' splice site to promote U12-type intron splicing and the second step of U2-type intron splicing". Pre-mRNA splicing plays a key role in gene expression and regulation in higher eukaryotes. Pre-mRNA splicing occurs in a large RNA-protein complex called a spliceosome. A spliceosome consists of the $\mathrm{U} 1, \mathrm{U} 2, \mathrm{U} 4, \mathrm{U} 5$, and U6 snRNP com- 
plexes and a large amount of proteins. Professor Shen presented experimental evidence that the U2A35-related protein Urp is involved in splicing the major class of U12-type introns. Pre-mRNA splicing is a stepwise process. In the first step, the $5^{\prime}$ splice site is cleaved, and then subsequently the $3^{\prime}$ splice site is cleaved; the two exons are then ligated together to form a mature RNA. Professor Shen presented experimental results suggesting that certain alternative splicing events regulate oncogenesis and tumor metastasis.

The second speaker of the first session was Dr. Kyoung Mi Kim from Korea University, Korea. Dr. Kim is a recipient of the prestigious post-doctoral grant from the President of Korea. In her talk, she summarized the hard work performed during her doctoral program. The title of her talk was "CTIF regulates replication-dependent histone $m R N A$ stability and translation". She began with the cap-binding proteins 80 and 20 (CBP80/20) and the translation initiation factor 4E (eIF4E). She then demonstrated that the stem-loop binding protein SLBP functions as a new CBP80/20-dependent translation initiation factor (CTIF)-interacting protein. CBP80/20-dependent translation is tightly coupled to nonsense-mediated mRNA decay (NMD), which was the next speaker's topic. The eukaryotic initiation factor 4GI (eIF4GI) was previously known as the initiator of both CBP80/20-dependent translation and eIF4E-dependent translation. Dr. Kim identified CTIF as a new initiator of CBP80/20-dependent translation (Kim et al., 2009). She found that CTIF directly interacts with CBP80, which is necessary for CBP80/20-dependent translation initiation. Furthermore, her group showed that CTIF directly interacts with eIF3g, which is a subunit of the eIF3 complex. The eIF3 protein complex is known to induce ribosomes to initiate translation. The interaction between CTIF and eIF3g provides evidence that CTIF actually works as an initiator of CBP80/20-dependent translation. Using yeast two-hybrid screening, her group is working to find new factors that interact with CTIF, which will give us a better understanding of the function of CTIF in CBP80/20-dependent translation.

The next speaker was Professor Jungwook Hwang from Hanyang University, Korea. The topic of his presentation was "Nonsense-mediated mRNA decay (NMD): mechanism and application". NMD is a particularly frugal way to regulate mRNA translation. NMD is a conserved mRNA surveillance mechanism that degrades premature termination codon (PTC)-harboring mRNAs, though the specific mechanisms are different among species. Transcription is a surprisingly sloppy process that creates PTCs through faulty splicing, DNA rearrangements, and point mutations. Professor Hwang estimated that NMD regulates as much as $35 \%$ of the alternatively spliced transcripts and $\sim 10 \%$ of all transcripts in humans. He presented experimental evidence that the interaction of up-frame shift factor 1 (UPF1) and cap-binding protein 80 (CBP80) is critical in NMD. To date, many NMD studies focused on the events after PTC recognition. Before PTCs are recognized by the ribosome, however, certain cellular signals can enhance NMD. SMG1 stability is promoted by casein kinase 2- (CK2-) mediated phosphorylation of telomere maintenance 2 (TEL2). Inhibition of CK2 activity and unphosphorylated TEL2 impair NMD, suggesting that cellular signaling regulates NMD activity. Altogether, Prof. Hwang's research suggests that NMD efficiency may be controlled by the modulation of cellular signaling (Hwang and Maquat, 2011).

On the second day of the meeting, four speakers presented their recent findings on RNA metabolism. The first speaker was Prof. Sung Wook Chi from Sungkyunkwan University, Korea. The title of his talk was "Genome-wide decoding of $m R N P$ and miRNA maps". Professor Chi introduced a genome-wide method to identify microRNA (miRNA) target sites and discussed a new rule of miRNA target recognition. Although miRNA regulation is important for generating the biological complexities underlying various phenotypes, our understanding of such regulation at the genome-wide level lags far behind that of transcriptional control, due to methodological restrictions. Therefore, Prof. Chi developed a genome-wide means of mapping RNA binding protein (RNABP) target sites in vivo by analyzing high-throughput sequencing (HITS) data from RNAs isolated by cross-linking immunoprecipitation (CLIP). Recently, the HITS-CLIP method was further applied to Argonaute (Ago) proteins; core proteins imparting miRNA-mediated repression. Ago HITS-CLIP tries to solve the problem of identifying miRNA target sites, which are hard to predict because miRNA activity only requires base pairing by six to eight seed nucleotides. By cross-linking native Ago-RNA complexes in mouse brain cells, Ago HITS-CLIP combined with bioinformatic analysis precisely identified miRNA-target interaction sites. Not all Ago-miRNA-mRNA interactions corresponded to known seed sequences, leading to the discovery of a new rule for miRNA-mRNA interactions: the "pivot-pairing rule", which describes the bulged pairing (positions 5-6) of target mRNAs to miRNA seed regions (Chi et al., 2012). The pivot pairing rule expands our ability to identify potential regulatory sites for miRNAs and provides insight into the biochemical mechanisms by which Ago-miRNA complexes bind their targets.

The second speaker was Prof. Jae Bok Heo from Dong-A University, Korea. His topic was plants, and the title of his talk was "Coordinated epigenetic silencing of FLC by long non-coding RNA in Arabidopsis". Beyond their beauty, flowers are extremely important because the survival of plants depends on successful flowering to ensure seed production for the next generation, and all life forms depend on the energy produced by plants via photosynthesis. Professor Heo presented evidence of the vernalization-mediated epigenetic silencing of the FLC gene by long noncoding RNAs in Arabidopsis. He explained that vernalization (cold winter conditions) triggers the enrichment of Tri-methylated Lys 27 in histone H3 (H3K27me3) 
in the chromatin of $F L C$, a potent floral repressor. The enrichment of $\mathrm{H} 3 \mathrm{~K} 27 \mathrm{me} 3$ results in epigenetically stable repression of FLC in Arabidopsis. Plant cells remember this experience and maintain the repression, resulting in a slower transition to flowering in the spring. The long intronic noncoding RNA COLDAIR, transcribed from the $F L C$ first intron, is required for the stable epigenetic repression of $F L C$ during the winter. COLDAIR is induced by cold temperatures and recruits polycomb repressive complex 2 (PRC2) to the FLC chromatin, thereby enriching $\mathrm{H} 3 \mathrm{~K} 27 \mathrm{me} 3$ during/after vernalization. COLDAIR was first characterized as a long noncoding RNA in plants. Thousands of long noncoding RNAs are transcribed in plants and regulated by many molecular signals, abiotic and biotic stresses, and several hormones (Heo and Sung, 2011).

The third speaker was Dr. Jong Heon Kim from the National Cancer Center, Korea. The title of his talk was "Let-7 microRNA turnover: role of novel RNA-binding protein and 3' to 5' exoribonuclease for the let-7 microRNA biogenesis". Dr. Kim began with a good introduction to microRNA biogenesis. He then focused his talk on the let-7 miRNA family, a prototype microRNA family in humans, and its tumor-suppressive role targeting various oncogenic transcripts. Expression of let-7 is regulated at the post-transcriptional level; the RNA-binding protein Lin28 and the 3' terminal uridyltransferase ZCCHC11 have major roles. Lin28 anchors the let-7 precursor (pre-let-7) and ZCCHC11 catalyzes the addition of a 3' oligomeric uridine tail. The RNA-binding protein HuR also plays a role as an interaction partner. The over-expression of HuR enhanced both precursor and mature let-7 levels, and knockdown of HuR reduced let-7 levels. PARN, a well-known poly(A)-specific ribonuclease, also showed previously unknown $3^{\prime}$ and $5^{\prime}$ exodeuridylase activity toward oligouridylated pre-let-7. HuR recruits all the components for both oligouridylation and deuridylation; Lin28, ZUCCH11, Dicer, and PARN. Thus, both ZCCHC11 and PARN are present in the same Lin28-HuR miRNP complex, and their opposing activities modulate the length of the oligomeric uridine tail in pre-let-7. All these data suggest that the post-transcriptional regulation of let-7 biogenesis is a complex and multi-directional mechanism, and that $\mathrm{HuR}$ and PARN are potentially critical regulators of let-7 biogenesis, including its tumorigenic steps.

The last speaker was Prof. Sunju Jeong from Dankook University, Korea. The title of her talk was " $R N A$-mediated mechanism for $\beta$-catenin protein accumulation by Wnt signaling". She began her talk with a good overview of the onset and progress of different types of cancer. She then pointed out the importance of RNA stability for translation efficiency. Canonical Wnt signaling controls cell-fate determination, cell proliferation, and stem-cell maintenance. The level of
ß-Catenin, a key oncogenic transcription factor involved in Wnt signaling, is kept low in normally differentiated cells via binding by the adenomatous polyposis destruction complex (APC) and post-translational proteolytic regulation in the cytoplasm. Upon Wnt stimulation, proteolysis of $\beta$-catenin is inhibited. $\beta$-catenin then accumulates and translocates to the nucleus, activating the transcription of many target genes. Aberrant activation of the Wnt signaling pathway is involved in cancer-cell initiation in colon epithelium and other tissues, and is known to be a major cause of colorectal and other epithelial cancers. In fact, deletion and frequent loss of the APC gene as well as mutations of $\beta$-catenin gene are reported in colon cancer cells. The mechanisms behind $\beta$-catenin activation and the transcription-related functions of $\beta$-catenin, however, are not completely understood. Professor Jeong reported her recent findings that $\beta$-catenin can directly bind RNA through central Armadillo-repeat domains. Specifically, she showed the biochemical and cell-biological evidence that ß-catenin recognizes specific RNA motifs in the 3' UTR of cyclooxygenase- 2 mRNAs, and thus regulates the stability of these mRNAs. These data suggest that $\beta$-catenin is an RNA-binding protein and that the RNA-related functions of ß-catenin may contribute significantly to tumorigenesis in colon-cancer cells. Professor Jeong also discussed a novel mechanism by which ß-catenin may regulate multiple layers of gene expression. She concluded by proposing new concepts and perspectives on the development of therapeutic cancer interventions that target the aberrant-RNA binding properties of ß-catenin.

\section{References}

Chi SW, Hannon GJ, and Damell RB (2012) An alternate mode of miRNA target recognition. Nat. Struct. Mol. Biol. 19: 321-327. Dennis C (2002) The brave new world of RNA. Nature 418: 122-124. Heo JB and Sun S (2011) Vernalization-mediated epigenetic silencing by a long intronic noncoding RNA. Science 331:76-79

Hwang J, and Maquat LE (2011) Nonsense-mediated mRNA decay (NMD) in animal embryogenesis: to die or not to die, that is the question. Curr. Opin. Genet. Develop. 21: 422-430.

Kim KM, Cho H, Choi K, Kim J, Kim BW, Ko YG, Jang K, and Kim YK (2009) A new MIF4G domain-containing protein CTIF directs nuclear cap-binding protein CBP80/20-dependent translation. Genes Dev. 23: 2033-2045.

Mattick J and Makunin IV (2006) Non-coding RNA. Hum. Mol. Genet. 15: R17-R29.

Shen H, Zheng X, Luecke S, and Green MR (2010) The U2AF35-related protein Urp contacts the 3 ' splice site to promote U12-type intron splicing and the second step of U2-type intron splicing. Genes Dev. 24: 2389-2394. 